\title{
The Alternative Cellular Energy (ACE) Pathway as the Foundation for a New Paradigm in Medicine
}

\section{Abstract}

Physicians currently regard most chronic illnesses as biochemical disorders that require long-term pharmaceutical therapy. While restoring biochemical balance in the diseased cells, pharmaceuticals can affect similar biochemical pathways in normal cells. As expected, this commonly leads to adverse clinical side effects. Given an adequate amount of energy, cells can potentially work at restoring their own normal functions. A major boost to energy-based medicine is the identification of an energy pathway in living organisms, which is distinct from the metabolism of food, and also distinct from photosynthesis in plants and in certain bacteria. The third or alternative cellular energy (ACE) pathway is expressed as a dynamic (kinetic) quality of the body's fluids. It arises from the attraction into the body of a natural environmental force termed KELEA (kinetic energy limiting electrostatic attraction). The administration and/or consumption of KELEA activated water can be used to enhance the body's ACE pathway, as may certain forms of electrical activity of the brain. This article summarizes studies performed on the ACE pathway and outlines the scope of potential clinical benefits of enhancing the ACE pathway as the primary therapy of many illnesses.

Keywords: ACE; Alternative cellular energy; ICE; Insufficient cellular energy; KELEA; Kinetic energy limiting electrostatic attraction; Calories; Waterceuticals ${ }^{\text {TM}}$; Activated Water; Enercel; Stealth adapted viruses
Review Article

Volume 9 Issue 6 - 2017

W John Martin*

Institute of Progressive Medicine, USA

*Corresponding author: $\mathrm{W}$ John Martin, Institute of Progressive Medicine, 1634 Spruce Street, South Pasadena CA, USA, Tel: (626) 616-2868; Email: wjohnmartin@ccid.org

Received: November 04, 2017 | Published: December 05, 2017
Abbreviations: ACE: Alternative Cellular Energy; ICE: Insufficient Cellular Energy; KELEA: Kinetic Energy Limiting Electrostatic Attraction; P: Phosphate; ADP: Adenosine Diphosphate; ATP: Adenosine Triphosphate; C: Centigrade; kg: Kilogram; CPE: Cytopathic Effect; CTL: Cytotoxic T Lymphocytes; UV: Ultraviolet; NMR: Nuclear Magnetic Resonance; ORP: Oxidation Reduction Potential

\section{Introduction}

It is widely assumed that the energy required for cellular functions in humans and animals is entirely derived from the metabolism of food [1]. Plants and certain bacteria can additionally obtain energy from sunlight for photosynthesis [2]. Specifically, by recycling the chemical rubisco, plants combine carbon dioxide with water to form carbohydrates and oxygen [3]. Cellular metabolism allows for the oxygen dependent reconversion of carbohydrates back to carbon dioxide and water, with the release of chemical energy. Much of the released chemical energy is utilized by cells to add a third phosphate (P) group to adenosine diphosphate (ADP) to form adenosine triphosphate (ATP) [4]. The reconversion of ATP to ADP plus P generates the chemical energy for many cellular functions, including the synthesis and functional activities of cellular components [5]. Synthesized components in plants and animals provide the food that is consumed and metabolized by other animals and humans [6].

The energy derived from food metabolism is expressed as calories or kilocalories (Calories). One Calorie is a measure of the amount of energy required to raise the temperature of a kilogram (kg) of water by $1^{\circ}$ Centigrade (C). Approximately 2,0002,500 Calories are consumed daily by regular adults [5-6]. For an individual weighing $75 \mathrm{~kg}$ and living in an environment with an overall average temperature of $17{ }^{\circ} \mathrm{C}$ and assuming that the body's heating is equivalent to that of water, then to reach normal body temperature of $37{ }^{\circ} \mathrm{C}$ will require $75 \times$ (37-17) Calories, i.e. 1,500 Calories. The body's temperature falls to that of the environment well within 24 hours following death. This means that the Calories that are needed to maintain body temperature are required daily. Given the daily work expenditures of skeletal, cardiac and smooth muscles, together with work performed by other organs and required to replace the energy lost in the excretion of urea, etc., the remaining $500-1,000$ daily Calories are seemingly inadequate. This issue has been largely neglected in biology. Some suggested answers include the possible role of melanin in energy delivery [7] and the potential release of energy in the theoretical transmutation of atoms, such as the conversion of potassium to calcium atoms [8].

\section{Alternative Cellular Energy (ACE) Pigments}

A further indication of an alternative cellular energy (ACE) pathway came from studies on stealth adapted viruses [9-12]. These viruses are not effectively recognized by the cellular immune system. This is because of the loss or mutation of the genes coding for the relatively few virus components normally targeted by cytotoxic T lymphocytes (CTL). Nevertheless, clinical recovery can occur in infected individuals and animals. Moreover, unless frequently re-fed, the cytopathic effects (CPE) caused by these viruses in tissue cultures undergo a somewhat remarkable 
recovery process [13]. The cellular recovery is associated with the production of energy-transducing materials by the infected cells. These materials can aggregate in the extracellular fluid medium into particles, threads, and ribbons. Their energy-transducing properties include fluorescence under both ultraviolet (UV) and visible light illumination, electrostatic attraction and repulsion and occasionally ferromagnetism [13]. Smaller particles in fluid medium display mobility well beyond that of Brownian movement. The larger particles can also donate electrons and generate vapor bubbles when placed in distilled water. Furthermore, the particles can lead to the abiotic synthesis of lipids in tissue culture medium $[13,14]$.

A striking feature of both in vivo and in vitro virus infected cells is the marked disruption of the cells' mitochondria $[15,16]$; the major site of energy production from the metabolism of food [17]. It was reasonably argued that the intra- and extracellular particles were providing an alternative source of cellular energy beyond that of mitochondria-based food metabolism. Since the particles were commonly pigmented, they were termed ACE pigments [13]. The addition of a few ACE pigment particles to fresh tissue culture medium prevented the reactivation of the virus $\mathrm{CPE}$, which can rapidly occur upon re-feeding of morphologically repaired stealth adapted virus infected cultures [13].

\section{Homeopathy as Activated Water}

The reactivation of stealth virus induced CPE was also prevented using a product that is marketed as a homeopathic remedy. The product is called HANSI (homeopathic activator of the natural system immune). Based on the author's in vitro finding, the United States manufacturer of HANSI renamed and has since improved the product, which is now called Enercel (www.enercel.com).

Classical homeopathy is based on the flawed assumption of specificity of action [18]. According to the Law of Similars [19], chemical compounds that induce specific symptoms when administered in large quantities to healthy individuals can alleviate the same symptoms when administered in minute dosages to patients exhibiting those same symptoms. It is even questioned whether there are any residual chemicals in many of the water-based homeopathic remedies; with the suggestion that the water may have acquired some form of specific memory of the chemicals used in formulating the product [20]. To the author's knowledge, no cross-over studies showing specificity of action of homeopathic formulations have been published. Indeed, products such as Enercel have shown clinical efficacy in many diverse clinical situations, including cancer, infections, asthma and neurodegenerative illnesses [21].

Water with Added Benefits in Medicine and in Agriculture

Belief in the healing properties of water preceded the practice of homeopathy. Water from certain locations in the world is especially viewed as effective in healing many aliments. The clinical benefits are generally attributed to special blends of various electrolytes or other "magical" components. Early pharmacies would market such water for their rejuvenating benefits. Interesting examples include Quinton Water obtained directly from plankton-rich, vortex swirling areas in the ocean [22]; and Geovital H3, a procaine containing formulation developed by Dr. Ana Aslan [23]. A much broader industry of marketing bottled water has subsequently emerged. In compliance with the Food and Drug Administration (FDA) and similar regulatory authorities in other countries, specific medical claims have been replaced with emphasis on taste with implied improved wellness and vitality from drinking the water.

Farmers have also believed in the superiority of water from certain locations in promoting the growth of various crops. Again, the benefits are usually attributed by the farmers to the mineral content of the water. Various additives have been developed as water and soil amendments. Some of these products go beyond supplying bioavailable minerals. For example, a tree sap distillate from Japan called HB-101 was shown by the author to consist of a complex array of terpenes and terpenoids (unpublished). Only five drops per gallon of water are required to significantly improve agricultural crop productivity and extending the shelf life of harvested produce (HB-101.com). Examples of products ostensibly supplying bioavailable minerals include humic and fulvic acids, zeolites, tourmaline, mica, crushed volcanic rock, shungite (a product from Russia), magnesium and other mineral oxides, water from the Great Salt Lake in Utah, etc.

The prospect of water being intrinsically improvable for human and animal consumption and also for agricultural purposes has been promoted by several developers. Mr. Johann Grander realized that a copper-rich water obtained from deep in an abandoned copper mine in Austria had beneficial properties. Moreover, even having regular water flow into a device with a closed inner compartment containing the beneficial water from the mine, would improve the performance of the water flowing out of the device, when tested in numerous agricultural and industrial applications (www.grander.com). Mr. Yul Brown showed that the gases evolving from water electrolysis could change regular water by simply being bubbled into the water [24]. In addition to hydrogen and oxygen, Brown's gas is now known to have a component of non-thermally vaporized water molecules. Many promoters have pursued the supposed benefits of hydrogen and/or hydroxyl ions in hydrogen-rich and alkali water, respectively. The hydrogen and/or hydroxyl ions can be generated by electrolysis, plasma fields, and metals, such as magnesium. Various metal halides can also be used. Similar to the questionable attribution of electrolyte-activated water to the consumption of electrolytes, the activating gases may be indirectly functioning by inducing intrinsic changes in the water. Thus, it is noteworthy that many of the same activating compounds that are normally added to water are able to affect water that is simply placed nearby to the compounds. These compounds, including crystals, mixed metal oxides and coils can also be embedded into insoluble resins and take the form of simple spheres or more complex shapes, such as pyramids. Water activating energy fields can also be established using pulsed or resonating magnetic, electrical and light impulses $[25,26]$.

\section{Water Activating Compounds and Energy Fields}

A review of the compounds that are typically used to activate water points to the potential importance of spatial molecular separation of positive and negative electrical charges. Thus, many 
of the compounds are dipolar, a feature easily observed with ACE pigments. Most of the water activating devices can also be regarded as emitters of fluctuating levels of positive and negative electrical charges. The question was asked of physicists as to why electrostatically attracted opposing electrical charges do not fuse with one another, leading to their presumed annihilation. The answer was essentially "We don't really know." Even the failure of electrical neutralization of charges on colloidal particles [27] or on the persisting electrostatic imprint on paper from prior writing [28] are not easily explained. Along with additional rationale, a hypothetical natural force was conceived by the author and tentatively termed KELEA (kinetic energy limiting electrostatic attraction). This force is likely to be equivalent to the "life energy" force proposed by others and variously called chi, prana, orgone, etc., $[29,30]$ The term KELEA is preferable since it can be related to a basic physical property of electrical charges. It was proposed that ACE pigments, which clearly show electrostatic properties, were formed by cells in response to insufficient cellular energy (ICE) and that they acted as a biological attractant for bringing KELEA into the cells. Support for this concept has come from observing the systemic and local production of ACE pigments in response to various illnesses.

\section{Detection of ACE Pigments}

The UV fluorescence of ACE pigments is significantly enhanced in the presence of certain dyes, including neutral red and acridine orange. Neutral red dye has been used in conjunction with UV illumination as a useful screening tool to detect ACE pigment particles in dried perspiration and attached to strands of hair in stealth adapted virus infected patients. Areas of more diffuse fluorescence at, and just beneath the skin, have been observed in stealth adapted virus infected patients, including children with autism. Neutral red induced fluorescence is not specific for stealth adapted viruses, since it is also readily observed in skin lesions caused by herpes simplex virus (HSV), herpes zoster virus (HZV) and human papillomavirus (HPV) [31,32]. The electrostatic movements of ACE particles have led some patients to report on being infested with living parasites. A diagnosis of Morgellons disease is being popularized in social media for this condition [33,34]. Dermatologists and psychiatrists more commonly refer to the condition as delusional parasitosis [35], since most patients also exhibit neuropsychiatric symptoms. The author attributes the neuropsychiatric symptoms in these patients to the probability of an underlying stealth adapted virus infection [36]. Other investigators point to molecular evidence of Lyme disease in some of these patients [37]. Bacteria sequences can apparently be incorporated into replicating stealth adapted viruses, leading to the designation of viruses with bacteria-derived sequences as viteria [38]. Atypical bacteria have been cultured from several stealth adapted virus infected patients, with preliminary evidence for recoverable viruses from the bacteria. This observation raises important public health issues regarding the transmission of stealth adapted viruses.

\section{The Brain as a Natural Antenna for KELEA}

Based on personal encounters with some individuals and on experiments performed in conjunction with laughing yoga classes, it is proposed that the fluctuating electrical activity of the brain can function as a natural antenna for KELEA [39]. This proposed function may also extend to the fluctuating electrical activity of muscles, including the heart. The brain and possibly muscles are, accordingly, viewed as primary drivers of the ACE pathway. In addition to directly benefiting brain and muscle cells, the ACE pathway provides overall added support to the body's biochemical reactions. It can also benefit the body by increasing the perfusion of blood and body fluids. The proposed KELEA antenna function of the brain is consistent with the many indications from psychology that the brain can play an important role in the maintenance of good health and in the recovery from illnesses. Suggested correlations of brain activity with good health include an emphasis on parasympathetic versus sympathetic activity, increased levels of gamma waves, improved sleep and humor. A positive feedback is envisioned whereby a heightened ACE pathway may improve the brain's capacity to attract KELEA. This proposal can be experimentally tested by assessing a person's capacity to increase the activity of externally placed water. Since the production of ACE pigments is considered a secondary adaptation to ICE, the detection of ACE pigments can also serve as a marker for an individual's deficiency in their ACE pathway.

\section{Activated Water}

The term "activated water" has been applied to water with two opposing types of basic changes. For most practitioners, the term implies water with reduced viscosity and a collection of additional characteristics that when tested yield consistent, although quantitatively variable findings. In contrast, water located nearby to various types of hydrophilic membranes, so-called vicinal or interfacial water has increased viscosity and possibly exists in a somewhat crystalline state with reduced numbers of hydrogen atoms [40]. The reported biological benefits of activated water do not occur with interfacial water.

Some of the altered physical and chemical characteristics of the biologically beneficial activated water are shown in Table 1. Prominent features are the reduced surface tension, lower freezing and boiling points and increased volatility. Various promoters have highlighted certain indirect properties of their water, probably designed to add to the selling power of their product. For example, more uniform and attractive crystallization upon freezing, as presented by the late Dr. Emoto [41], is more appealing than simply reporting that the water freezes slower because of a lower freezing point.

${ }^{17} \mathrm{O}$ nuclear magnetic resonance (NMR) of activated water shows a reduced width of realignment [42]. This suggests that the presumed cluster formations by groups of water molecules are somewhat smaller in activated water (microclusters). Possibly related to this finding is the reported residual thread-like structures seen by scanning electron microscopy of dried "Double Helix" water [43]. Reduced viscosity and greater permeability of activated water are also consistent with the greater internal motions and faster dissolving patterns of particles of various dyes sprinkled onto the water.

An activated water product termed Neowater [44] was reportedly prepared using bismuth titanate nanoparticles as the activating compound (personal communication). Neowater was 
promoted as an effective means of increasing the performance of enzymes, including DNA polymerases, thereby, allowing for far less use of the enzyme [44]. This finding is directly relevant to the concept that more activated intracellular water can help promote intracellular metabolism. Even more significant is the apparent abiotic synthesis of lipids in fluids containing ACE pigments $[13,14]$. This finding is interpreted as a direct relationship between KELEA and chemical energy [45].

Table 1: Some of the Altered Characteristics of Activated Water*.

\begin{tabular}{|c|c|}
\hline Increased & Reduced \\
\hline Volatility & Surface tension \\
Permeability & Boiling \& Freezing points \\
Cellular hydration & Specific heat \\
Vortex upon spinning & Density \\
Diffusion of chemicals & Cohesion \& Viscosity \\
Internal movements & ORP\# level \\
Support of enzymes & Solubility of polar solutes \\
Cleaning action & Miscibility with ethanol \\
Infrared absorption & Scale formation \& Corrosion \\
Infrared emission & Width of NMR realignment \\
Abiotic lipid synthesis & Bacteria growth \\
\hline
\end{tabular}

*Water activation is a quantitative measure of an inducible change in water. Varying levels of activation are achieved using different methods. The above listing is a compilation of changes, which are more readily detected in relatively highly activated water.

\#ORP refers to Oxidation Reduction Potential. Reduced (negative) values of activated water indicate the capacity to donate electrons.

${ }^{\pi}$ Nuclear Magnetic Resonance of ${ }^{17} \mathrm{O}$ isotope of oxygen is interpreted as a measure of the size of cluster formations within the water.

\section{KELEA and Chemical Energy}

The potential chemical energy of a molecule is expressed as the amount of heat produced upon its combustion to simple gases plus water. Chemical reactions involve the conversion of one or more beginning molecules, referred to as reactants, to one or more different molecules, referred to as products. In order for a chemical reaction to occur, the total amount of chemical energy in the reactants, plus any externally supplied energy, must be greater than the total amount of chemical energy in the products of the reaction. The difference in the chemical energies between reactants and products is either released as heat or transferred to another chemical reaction.

Molecules comprise specific groupings of atoms that are linked together by chemical bonds. Unbound atoms are thought to yield some of their free energy as they undergo bonding with other atoms or groups of atoms. The yielded energy is commonly expressed as the bonding energy, as if the energy were stored within the chemical bonds. Indeed, some external energy is required to break chemical bonds. What is less certain is whether the amount of energy in the bonds is equivalent to the energy lost from the bonded atoms. Nor is it explained, how the released energy from the breaking of the bonds is returned to the freed atoms or groups of atoms.

An analogy exists between potential electrical and potential chemical energy. The potential electrical energy of a pair of opposite electrical charges is inversely related to the distance between the two charges. Some of this potential electrical energy is yielded as the distance between the two charges is reduced, that is, as the charges become more bound to one another. The charges regain their earlier energy if they are re-separated to their original distance from one another. KELEA is considered as a natural force that is primarily attracted to separated electrical charges. A proposed fundamental role for KELEA is to prevent the fusion and presumed annihilation of electrostatically attracted opposite electrical charges. Opposite electrical charges could still yield some, but not all, of the bound KELEA as they approach one another and regain the lost KELEA from the environment as they again re-separate from each other. A similar process could occur with atoms or groups of atoms as they separate from one another.

Support for equating KELEA with chemical energy [45] has been obtained in studies on KELEA activated gasoline and diesel fuels $[46,47]$. Various methods have been empirically described as improving the efficiency of fuel utilization. These methods run parallel with the methods used to activate water and, therefore, presumably lead to increases in the levels of KELEA in the fuels. Part of the benefit of using KELEA activated fuels arises from more complete combustion, with less unburnt fuel and carbon monoxide production. The observed increases in vehicle mileage using KELEA activated fuels extends well beyond the reduction in residual unburnt fuel. Indeed, the data seemingly indicate that KELEA increases the intrinsic chemical energy of the fuel $[46,47]$.

Metabolic processes are commonly interrelated, such that chemical energy from one chemical reaction is used to drive a closely linked different chemical reaction. While heat is acknowledged as an output of some chemical reactions, it is not perceived as an effective means to transfer energy between chemical reactions. One investigator has suggested that water can cluster into either low- or high-density domains, with the latter doing so with the input of chemical energy [48]. Low-density water domains are then presumed to act as carriers of chemical energy, reverting to high-density domains upon the transfer of their added energy. This type of scenario is equally compatible with the notion that KELEA acts as the transferrable energy between chemical reactions [45].

\section{Membrane Depolarization as the Initial Input of KELEA into Developing Life Forms}

As noted earlier, ACE pigments can mediate the abiotic synthesis of lipids. The appearances of the lipids include long trough shaped needles, solid crystals, pyramid shaped structures and broad membranes [13,14]. The evolutionary development of living cells required the formation of membranes, which are intrinsically impermeable to certain cations and/or anions. Lipid membranes can enclose spaces in which electrical differences 
exist between the inside and the outside of the enclosed spaces. Any event which allows for the passage of electrical ions across the membrane will hypothetically lead to the release of some of the KELEA bound to the separated electrical charges. The released KELEA can then presumably be used as chemical energy for the further synthesis of components within the enclosed space. Arguably, this process has preceded the membrane partitioning of hydrogen ions, as occurs in photosynthesis [2] and in ATP production from the metabolism of food [5].

Membrane depolarization is a primary feature of nerve and muscle cells. It was suggested, therefore, that depolarization may be a primary source of cellular energy for neurons $[39,49]$. Accordingly, neuronal cell hyperactivity may be an adaptive response of neuronal cells to ICE. As discussed elsewhere, hyperactivity is not a sustainable adaptation and energy deficient cells can enter into a quiescent phase [49]. Although the cells are still viable, they may lack the energy required to perform more specialized functions. As discussed later, both hyperactivity and hypo-activity can potentially be corrected via the ACE pathway and potentially from the consumption of KELEA activated water.

\section{Clinical Applications of KELEA Activated Water}

The most apparent therapeutic uses of KELEA activated water is for medical conditions in which the metabolism of food is impaired. This can result from insufficient oxygen because of diseases such as chronic obstructive pulmonary disease (COPD); impaired blood supply from cardiovascular and cerebrovascular diseases; metabolic derangements, for example in diabetes and malnutrition; and increased energy demands as in infections and during tissue repair $[50,51]$.

The ACE pathway compares favorably with the immune system in the defense against infectious diseases. Neutral red with the body's own ACE pigments or when added to certain KELEA activated fluids is very effective in UV phototherapy in expediting the healing of skin lesions caused by HSV, HZV, and HPV $[31,32]$. The UV illumination of KELEA activated fluids with dissolved neutral red dye is also beneficial in treating patients with presumed infection with stealth adapted viruses. Specifically, UV illumination of the fluid applied close to, but not in direct contact with the child's skin, achieved major improvements in children with autism [52]. Injection plus inhalation of Enercel led to significant suppression of both HIV and tuberculosis in patients with AIDS [53]. In another study, Enercel was shown to be effective in treating multi-drug resistant tuberculosis [54]. Two intramuscular injections of Enercel were shown in a controlled study to be effective in suppressing tropical diarrhea in young children [55].

A major downside of the immune system is its capacity to evoke tissue-damaging inflammation. Enercel is anti-inflammatory, as shown by its effectiveness in alleviating asthma, atopic dermatitis and even skin blemishes, such as cellulite. Burns and other types of trauma; chronic infections; and ischemia resulting in tissue damage will typically evoke an inflammatory response that can lead to disfiguring and functionally impairing scarring of the skin. Directly applying Enercel or copper-silver-citrate (CSC) activated water to acute burns can greatly suppress the inflammatory reaction and lead to complete healing without scarring [56].
KELEA activated fluids should be available to all first responders to fires. The fluids can be applied as a spray, used to moisten skin covering materials and also administered systemically. Still open for investigation is the emergency use of KELEA activated water in preventing organ scarring from myocardial infarctions and strokes.

Many cancers can be explained as a failure of genetically altered cells to undergo apoptosis, an energy requiring process. Cells undergoing apoptosis do not elicit inflammation. Both HANSI and Enercel have long histories in the therapy of cancers. Consistent with the suggestions that the added cellular energy provided by these products is being utilized for apoptosis is that the resulting tumor regressions are largely painless $[21,57,58]$. This is in contrast to chemotherapy, radiation therapy and immunotherapy, which are commonly accompanied by significant local pain. ACE pathway-based therapy should probably be tried as first-line therapy in all non-operable cancers.

A major category for ACE pathway-based therapy involves the restoration of normal neuronal cell functions. As noted above, hyperactivity as well as hypo-activity of neurons can be consequences of ICE. Neuronal cells regulate a vast array of functions within the brain and elsewhere in the body. Indeed, pinpointing certain illnesses to a specific biochemical disorder can overlook the basic failure of the brain to establish compensatory mechanisms to counter the disorder. The brain is directly involved in sensory, motor and autonomic nervous system activities, with input from various neurosensory organs that convey information relating to the environment. The brain also controls emotional moods and cognitive functions. Sleep is regarded as a critical factor in sustaining optimal brain activity. The proposed reduced viscosity and greater permeability of KELEA activated body fluids are likely to be beneficial in the removal of metabolic waste products from the brain, which is thought to especially occur during sleep.

Mental illness is a major tragedy for those directly affected, as well as those indirectly affected. So too are neurodegenerative illnesses that are probably better called neuro-quiescent illnesses, including Alzheimer's disease. Stealth adapted viruses can contribute to the ICE of neuronal cells in many of these illnesses $[49,59,60]$. There is an urgent need to include such patients into well controlled clinical studies using ACE pathway based therapies. It is particularly noteworthy that cancer and infectious disease patients who have been treated with various modalities to improve their ACE pathway, including the consumption, inhalation or direct skin exposure to KELEA activated water, have reliably reported on improved mood, self-esteem, and cognition. There have also been dramatic restorations of long-impaired sensory functions, including improved eyesight, hearing and taste, following short-term ACE pathway based therapy (unpublished clinical observations). These improvements have persisted beyond the specific treatment periods. These clinical observations support the tentative assessment that, in addition to improving a specific illness, enhancing the ACE can assist in the proposed KELEA antenna function of the brain. The potential capacity is for short-term, easily applied, ACE pathway enhancing therapy to achieve sustainable improvements in overall health. This approach contrasts with the pharmaceutical model that requires life-long, specific drug-based therapies for many illnesses. 


\section{Conclusion}

The prospect is emerging for a near-universal non-toxic "waterceutical ${ }^{\mathrm{TM} \text { " }}$ therapy for many of the major illnesses affecting mankind. It can reasonably be assumed that an optimal ACE pathway will also help in the prevention of many of the illnesses discussed in this paper. A three-phase approach is envisioned to further develop this new paradigm in the therapy and prevention of illnesses. The most important phase is the documentation of efficacy in well-conducted clinical studies. These studies can directly evaluate and compare different protocols in the treatment of a wide range of illnesses. The second phase is to ensure the availability of high quality KELEA activated water or other suitable methods necessary to conduct the clinical trials and, thereafter, to be available for widespread use throughout the community. The third phase involves gaining a better understanding of KELEA and the ACE pathway. A brief summation of some of the currently proposed properties and functions of KELEA are listed in Table 2. The collaboration of physicists, chemists and biologists with access to advanced instruments and technologies will lead to a better understanding of KELEA. These advanced studies will also test the possible relevance of KELEA to such major topics in science, such as global warming [61], cold fusion [62], wave-particle duality [62], gravity [63], etc. Useful practical and scientific input will also be provided by the concurrent applications of KELEA activated water to agriculture and industry [64-67].

Table 2: Properties and Functions of KELEA - A Basic Force of Nature.

\author{
Reversibly attracted to increasing positive and negative electrical charges \\ Partially released from decreasing positive and negative electrical charges \\ Primary purpose is to prevent the fusion of opposing electrical charges \\ Possibly explains the repulsion between identical electrical charges \\ Reduces the strength of inter-atom and intermolecular electrostatic bonding \\ Increases the intrinsic, dynamic (kinetic) activity of fluids, including water \\ Facilitates the catalytic activity of enzymes \\ Contributes to the energy available for chemical reactions \\ Can potentially lead to the prevention and cure of many illnesses \\ Can lead to abiotic synthesis or organic molecules \\ May have initiated the development of life \\ Contributes to the combustion energy of fuels \\ Is a source of cellular energy distinct from that provided by metabolism of food \\ Distinct from the cellular energy provided by sunlight in photosynthesis \\ Is potentially involved in several otherwise unexplainable natural phenomena
}

\section{Acknowledgement}

The Institute of Progressive Medicine is a component of MI Hope Inc., a non-profit public charity. Clinicians interested in testing KELEA activated water and KELEA generating medical devices may wish to directly contact the author. So too, may basic researchers wanting to assist in studies on KELEA.

\section{Conflict of Interest}

None.

\section{References}

1. Blaxter KL (1989) Energy Metabolism in Animals and Man. Cambridge University Press, Cambridge, USA, pp. 339.
2. Pessarakli M (2016) Handbook of Photosynthesis. ( $3^{\text {rd }}$ edn), CRC Press, Boca Raton, Florida, UK, pp. 846.

3. Anderson I, Backlund A (2008) Structure and function of rubisco. Plant Physiol Biochem 46(3): 275-291.

4. Yoshida, M, Muneyuki E, Hisabori T (2001) ATP synthase- A marvelous rotary engine of the cell. National Review Molecular Cell Biology 2(9): 669- 677.

5. Patton KT, Thibodeau GA (2016) Nutrition and Metabolism. ( $9^{\text {th }}$ edn), Chapter 41, In Anatomy \& Physiology, Elsevier, Amsterdam, Netherlands, pp. 930-965.

6. Coultate T (2015) Food. The Chemistry of its Components. ( $9^{\text {th }}$ edn), Royal Society Chemistry, Cambridge, UK, pp. 599. 
7. Goodman G, Bercovich D (2008) Melanin directly converts light for vertebrate metabolic use: heuristic thoughts on birds, Icarus and dark human skin. Med Hypotheses 71(2): 190-202.

8. Kervran CL, Abehsera M (1972) Biological Transmutations, and Their Applications in Chemistry, Physics, Biology, Ecology, Medicine, Nutrition, Agriculture, Geology. Swan House Publishing, Brooklyn, New York, USA.

9. Martin WJ, Zeng LC, Ahmed K, Roy M (1994) Cytomegalovirus-related sequences in an atypical cytopathic virus repeatedly isolated from a patient with the chronic fatigue syndrome. Am J Pathol 145(2): 441452.

10. Martin WJ (1994) Stealth viruses as neuropathogens. CAP Today 8(10): 67-70.

11. Martin WJ, Ahmed KN, Zeng LC, Olsen JC, Seward JG, et al. (1995) African green monkey origin of the atypical cytopathic "stealth virus" isolated from a patient with chronic fatigue syndrome. Clin Diagn Virol 4(1): 93-103.

12. Martin WJ (2014) Stealth adaptation of viruses: Review and updated molecular analysis on a Stealth adapted African green monkey simian cytomegalovirus (SCMV). J J Hum Virol Retrovirol 1(4): 00020.

13. Martin WJ (2003) Stealth virus culture pigments: A potential source of cellular energy. Exp Mol Pathol 74(3): 210-223.

14. Martin WJ (2014) The alternative cellular energy (ACE) pathway in the repair of the cytopathic effect (CPE) caused by stealth adapted viruses: In vitro and in vivo evidence supporting a new therapeutic paradigm. In Stealth Adapted Viruses; Alternative Cellular Energy (ACE) \& KELEA Activated Water. Author House, Bloomington, Indiana, USA, p. 31-70.

15. Martin WJ (1996) Severe stealth virus encephalopathy following chronic fatigue syndromelike illness: Clinical and histopathological features. Pathobiology 64(1): 18.

16. Martin WJ (2003) Complex intracellular inclusions in the brain of a child with a stealth virus encephalopathy. Experimental \& Molecular Pathology 74(3): 179-209.

17. Scheffler IE (2011) Mitochondria. ( $2^{\text {nd }}$ edn), John Wiley \& Sons, Hoboken, New Jersey, USA, pp. 480.

18. Marcy EE, Hunt FW (1868) The Homeopathic Theory and Practice of Medicine. William Radde publisher, New York, USA, pp. 942.

19. Gutman W (1961) The basis of the law of similars. British Homoeopathic Journal 50(1): 26-29.

20. Consigli P (2008) Water, Pure and Simple: The Infinite Wisdom of an Extraordinary Molecule. Watkins Publishing, London, pp. 400.

21. Martin WJ, Laurent D (2015) Homeopathy as a misnomer for activation of the alternative cellular energy pathway: Evidence for the therapeutic benefits of Enercel in a diverse range of clinica illnesses. Int J Complement Alt Med 2(1): 00045.

22. Dittman R, Brugioni R (2006) Evolutionary development of our internal ocean: Restoring bio-terrain with Quinton marine plasma. Explore 15(6): 1-5.

23. Peris $T$ (2013) The reappearance of procaine hydrochloride (Gerovital H3) for antiaging. Journal of the American Geriatrics Society 61(6): 1024-1025.

24. Hurtak JJ, Hurtak D (2014) The history and future of Brown's gas. Nexus Magazine 21(4): 45-54.

25. Martin WJ (2015) Interacting light paths attract KELEA (kinetic energy limiting electrostatic attraction) and can lead to the activation of water. Open Journal of Biophysics 5: 115-121.
26. Martin WJ (2015) Interactive electric fields can attract KELEA (kinetic energy limiting electrostatic attraction) and can lead to the activation of water. International Journal Complementary \& Alternative Medicine 1(6): 00034

27. Hidalgo-Alvarez R (2009) Structure and Functional Properties of Colloidal Systems. CRC Press, Boca Raton, Florida, USA, pp. 518.

28. Allen MJ (2015) Foundations of Forensic Document Analysis: Theory and Practice. John Wiley \& Sons, Hoboken, New Jersy. USA, pp. 272.

29. Rosch PJ (2009) Bioelectromagnetic and subtle energy medicine: the interface between mind and matter. Ann N Y Acad Sci 1172: 297311.

30. Reich W (1948) The Cancer Biopathy. The Discovery of the Orgone. In: White A,\& Higgins M, et al. (Eds.), Farrar, Straus and Giroux, New York, USA, pp. 433

31. Martin WJ, Stoneburner J (2005) Symptomatic relief of herpetic skin lesions utilizing an energy-based approach to healing. Experimental and Molecular Pathology 78(2): 131-134.

32. Martin WJ, Stoneburner J (2014) Alternative cellular energy (ACE) pathway activation as the mode of action of neutral red dye phototherapy of human viruses. J HumVirol \& Retrovirol 1(4): 00019.

33. Savely VR, Stricker RB (2007) Morgellons disease: The mystery unfolds. Expert Review Dermatology 2: 585- 591.

34. Middelveen MJ, Rasmussen EH, Kahn DG, Stricker RB (2012) Morgellons disease: A chemical and light microscopic study. Journal Clinical Experimental Dermatology Research 3: 140.

35. Al-Imam AML (2016) A systematic literature review on delusional parasitosis. Journal Dermatology \& Dermatologic Surgery 20(1): $5-14$.

36. Martin WJ (2005) Alternative cellular energy pigments mistaken for parasitic skin infestations. Exp Mol Pathol 78(3): 212-214.

37. Middelveen MJ, Stricker RB (2016) Morgellons disease: a filamentous borrelial dermatitis. Int J Gen Med 9: 349-354.

38. Martin WJ (1999) Bacteria related sequences in a simian cytomegalovirus-derived stealth virus culture. Experimental Molecular Pathology 66(1): 8-14.

39. Martin WJ (2015) Is the brain an activator of the alternative cellular energy (ACE) pathway? Int J Complement \& Alt Med 1(1): 00002.

40. Polack GH (2013) The Fourth Phase of Water: Beyond Solid Liquid and Vapor. Ebner and Sons, Seattle WA, Washington, USA, pp. 357.

41. Emoto M (2011) The Hidden Messages in Water. Atria Books, New York, USA, pp. 200.

42. Sun S, Wei CD, Liu YX (2010) Characterization and water activation behavior of tourmaline nanoparticles. Journal Nanoscience Nanotechnology 10(3): 2119-2124.

43. Gann DL, Lo SY (2009) Double Helix Water. Mass Market Paperback pp. 316.

44. Gabbai E (2006) Solid-fluid composition. United States Patent Application US 11/324,586.

45. Martin WJ (2017) Is KELEA (kinetic energy limiting electrostatic attraction) A source of chemical energy? MOJ Bioorganic \& Organic Chemistry 1(2): 00011.

46. Martin WJ (2016) KELEA (kinetic energy limiting electrostatic attraction) can markedly improve the performance of gasoline and diesel fuels in power generation and in transportation. Journal of Transportation Technologies 6(3): 148-154. 
47. Martin WJ (2017) Using KELEA (kinetic energy limiting electrostatic attraction) to improve the efficiency of fuel consumption. Open J Air Pollution 6(3): 103-116.

48. Wiggins P (2008) Life depends upon two types of water. PLoS One 3(1): e1406.

49. Martin WJ (2017) Hyper-excitability followed by functional quiescence in neuronal cells caused by insufficient cellular energy (ICE): Treatable by enhancing the alternative cellular energy (ACE) pathway. World Journal Neuroscience 7(3): 257-266.

50. Martin WJ (2015) Alternative cellular energy pathway therapy using KELEA activated water. Int Complement \& Alt Med 2(2): 00051.

51. Martin WJ (2016) Deconstructing medicine. The alternative cellular energy pathway. British Journal Medicine Medical Research 11(8): $1-6$.

52. Martin WJ (2014) Alternative cellular energy (ACE) pathway as natural therapy for autism. In Stealth Adapted Viruses; Alternative Cellular Energy (ACE) \& KELEA Activated Water. Author House, Bloomington, Indian, USA, p. 87-102.

53. Dubrov V, Dubrova T, Christner D, Laurent D, Martin WJ (2015) Alternative cellular energy based therapy using Enercel ${ }^{\mathrm{TM}}$ in advanced AIDS patients co-infected with tuberculosis and treated in Chernigov, Ukraine. J HumVirol \& Retrovirol 2(6): 00061.

54. Dubrov V, Dubrova T, Suhareva V, Christner D, Baiamonte J, et al. (2012) Efficacy of three weeks treatment with Enercel® for new onset, presumed drug-sensitive and confirmed multi-drug resistant pulmonary tuberculosis at the Regional Anti-Tuberculosis Hospital in Chernigov, Ukraine. Tuberculosis, Pulmonary Disease, HIV Infection 1: 85.

55. Izaguirre RR, Guzman MR, Fuentes RC, Mena CE, Penate E, et al (2014) Alternative cellular energy based therapy of childhood diarrhea. in Stealth Adapted Viruses; Alternative Cellular Energy (ACE) \& KELEA Activated Water. Author House, Bloomington, Indian, USA, pp. 103-112.

56. Martin WJ (2017) Tissue regeneration without scarring achieved by enhancing the alternative cellular energy (ACE) pathway. Journal of Cosmetics, Dermatological Sciences and Applications 7(1): 82-98.
57. Martin WJ (2016) Cancer as an Insufficiency of Cellular Energy (ICE): Therapeutic Approaches Based on Enhancing the Alternative Cellular Energy (ACE) Pathway. Int J Complement \& Alt Med 3(3): 00074.

58. Martin (2018) Cancer is Treatable via the Alternative Cellular Energy (ACE) Pathway. Submitted.

59. Martin WJ (205) Stealth adapted viruses. A bridge between molecular virology and clinical psychiatry. Open Journal Psychiatry 5(4): 311-319.

60. Martin WJ (2015) Stealth adapted viruses - Possible drivers of major neuropsychiatric illnesses including Alzheimer's disease. J Neurol Stroke 2(3): 00057.

61. Martin WJ (2016) KELEA, cosmic rays, cloud formation and electromagnetic radiation: Electropollution as a possible explanation for climate change. Atmospheric and Climate Sciences 6(2): 174-179.

62. Martin WJ (2016) KELEA (kinetic energy limiting electrostatic attraction) offers an alternative explanation to existing concepts regarding wave-particle duality, cold fusion and superconductivity. Journal of Modern Physics 7(15): 1995-2007.

63. Martin WJ (2016) KELEA (kinetic energy limiting electrostatic attraction) may add to the measured weight of an object. Journal of Modern Physics 7(6): 461-472.

64. Martin WJ (2015) KELEA activated water leading to improved quantity \& quality of agricultural crops. Adv Plants Agric Re 2(1): 00033.

65. Martin WJ (2015) Improved efficiency of heat exchange using KELEA activated water. Open Journal of Energy Efficiency 4(2): 36-43.

66. Martin WJ (2015) KELEA: A natural energy that seemingly reduces intermolecular hydrogen bonding in water and other liquids. Open Journal of Biophysics 5(3): 69-79.

67. Martin WJ (2015) KELEA activation of water and other fluids for health, agriculture and industry. Journal of Water Resource and Protection 7(16): 1331-1344. 